\title{
CONSENTIMIENTO INFORMADO EN ODONTOLOGÍA. UN ANÁLISIS TEÓRICO-PRÁCTICO
}

\author{
Leonor Palomer R.*
}

\begin{abstract}
Resumen: Para conocer acerca de la petición de consentimiento informado en odontología, se realizó una búsqueda de información en las bases de dato de SciELO y Pub Med, usando las palabras claves "consentimiento informado", "odontología" y "autonomía”. Como complemento, para tener información sobre la situación en Chile, se envió una encuesta a 40 cirujanos dentistas de tres centros de atención dental de la Red de Salud de la Pontificia Universidad Católica de Chile.
\end{abstract}

Se puede observar que aún existe desinformación en odontología en cuanto a la finalidad, implicancia e importancia de un adecuado proceso de consentimiento informado en la relación odontólogo-paciente.

Palabras clave: consentimiento informado, autonomía, odontología

\section{INFORMED CONSENT IN ODONTOLOGY. A THEORETICAL-PRACTICAL ANALYSIS}

Abstract: In order to know more about the petition of informed consent in odontology, we realized a search of information in the database of SciELO and Pub Med, using the key words "informed consent", "odontology" and "autonomy". As a complement, in order to have information about the situation in Chile, a survey was sent to 40 dental surgeons in 3 dental centers belonging to the health network of the Pontificia Universidad Católica de Chile.

It can be observed that there still exists disinformation in odontology in Chile relating to the purpose, implications and importance of an adequate process of informed consent in the dentist-patient relationship.

Key words: informed consent, autonomy, odontology

\section{O CONSENTIMIENTO INFORMADO EM ODONTOLOGIA: UMA ANÁLISE TEÓRICO- PRÁTICO}

Resumo: Para conhecer a respeito do pedido de consentimento informado em odontologia, realizou-se uma busca de informação na base de dados SciELO e Pub Med, usando as palabras chaves "consentimento informado", "odontologia” e "autonomia". Como complemento, para se ter uma informação sobre a situa;cáo no Chile, foi enviado um questionário para 40 cirurgiōes dentistas de trës centros de cuidados dentais da Rede de Saúde da Pontifícia Universidade Católica do Chile. Pode-se observar que ainda existe desinformação em odontologia relacionadas com a finalidade, implicância e importância de um adecuado processo de consentimento informado na relaçáo odontólogo-paciente.

Palavras chave: consentimiento informado, autonomia, odontologia

\footnotetext{
* Cirujano Dentista. Profesor Asistente de la Facultad de Medicina, Carrera de Odontología, Pontificia Universidad Católica de Chile, Chile
} Correspondencia: lpalomer@puc.cl 


\section{Introducción}

Actualmente, en el marco de la relación médico-paciente, se ha dado énfasis al principio de autonomía, entendido como el derecho del paciente a ser reconocido como persona libre y dueña de tomar sus decisiones. En este contexto aparece el concepto de "consentimiento informado", según el cual toda persona debe recibir, en términos comprensibles, una información completa respecto del diagnóstico, tratamiento y pronóstico de su enfermedad por parte del profesional de la salud. Este debe además conocer las implicancias en caso de rechazar el tratamiento y asumir las consecuencias tanto del éxito como del fracaso del mismo, junto con su tratante. También debe ser informado sobre los costos de su atención y la existencia de tratamientos alternativos.

El modelo clásico de atención paternalista, en el que el profesional toma las decisiones y actúa según su criterio, sin considerar la opinión de su paciente, ha evolucionado hasta nuestros días en que se incorpora al enfermo en el proceso de definir las acciones a seguir y se lo hace corresponsable de su tratamiento.

Este cambio se debe, en primer lugar, a un reconocimiento de los derechos de la persona y a la consideración de sus expectativas y necesidades, esto es, al creciente respeto por la autonomía del paciente. También existe la necesidad de contrarrestar una medicina tecnificada y poco humana, ya que se ha demostrado que la buena relación médico-paciente ayuda a la adhesión a los tratamientos y por lo tanto a su éxito. Además, los problemas médico legales han ido en aumento, lo que hace necesario usar un documento que demuestre la responsabilidad compartida de los pacientes en las decisiones tomadas.

Para que el consentimiento informado sea válido debe cumplir con ciertas condiciones(1):

- Debe entregarse toda la información médica adecuada para que el paciente tome su decisión suficientemente informado.

- El paciente debe entregar su consentimiento en forma voluntaria, sin presiones de ningún tipo.

- El paciente debe ser competente y/o capaz de tomar una decisión que puede afectar su salud. Si es menor de edad o no tiene capacidad para decidir, debe ser representado por sus padres o su representante legal.
- Que sea el mismo sujeto del acto médico quien lo otorgue (concepto de titularidad).

- Que el propósito y la causa del consentimiento sean recuperar la salud del enfermo.

- Que el proceso tenga una forma externa (verbal o escrita).

- Que el consentimiento sea obtenido previo a la realización del acto médico.

Estos conceptos, ya vigentes en la relación médico-paciente, son también aplicables a la relación odontólogopaciente, en especial considerando que el ejercicio de la odontología se realiza bajo condiciones cada vez más complejas. Actualmente existen mayores adelantos para llegar al diagnóstico y opciones terapéuticas que hace pocos ańos. Se cuenta con la posibilidad de utilizar materiales, técnicas y especialidades nuevas. Si bien esto ha significado mejores alternativas de tratamiento para el paciente y mejoría en su calidad de vida, también ha traído un incremento en la aparición de efectos adversos, complicaciones, fracasos de tratamiento y elevación de los costos.

El odontólogo trabaja en un territorio anatómico donde se realizan las más diversas funciones, algunas de ellas vitales, tales como respirar, hablar, deglutir, masticar, degustar.

Aunque ha habido avances significativos, todavía no es posible colocar estructuras dentarias naturales donde antes las había y los tratamientos consisten fundamentalmente en instalar artificios tecnológicos que deben adaptarse a las condiciones fisiológicas, lo que no siempre se logra. El porcentaje de fracasos puede ser mayor de lo que se espera y es importante que el paciente lo sepa. Si bien esto se puede explicar en términos de porcentaje de éxitos o fracasos, basado en lo reportado en la literatura, hay que hacer ver al paciente que la realidad es extremadamente variable y lo publicado no es más que una referencia. El hecho de traspasar al enfermo parte de esta información mejora sustancialmente la relación profesional-paciente.

Muchas veces la atención dental genera ansiedad y temor al dolor. El paciente se siente disminuido al estar en una posición semi-acostada en el sillón frente al odontólogo y éste suele utilizar términos técnicos y científicos de difícil comprensión. Si la patología produce dolor, el paciente puede estar dispuesto a aceptar un tratamiento sin tener real entendimiento de lo que le ofrecen. El profesional debe tomar el 
tiempo suficiente para explicar cuál es el problema, las opciones de tratamiento, sus costos y los riesgos que implican. Una conversación estando el odontólogo y el paciente sentados al mismo nivel, si hace falta usando diagramas, que expliquen con más claridad lo que se propone, asegurándose que el paciente entiende, es el primer paso para una relación participativa, la que le permite involucrarse voluntaria, consciente y activamente en la adopción de decisiones respecto del tratamiento de su salud.

Es importante el uso de formularios en los que el paciente puede leer lo que se le ha planteado y firmar su consentimiento, con el fin de registrar y monitorear, y para probar legalmente que se dio la información y se obtuvo el consentimiento(2).

Jorge Triana Estrada(3), director de Estomatología de la Comisión Nacional de Arbitraje de México, advierte que en su país las irregularidades con mayor incidencia y frecuencia son, en primer lugar, la falta de comunicación entre el profesional de la salud bucal y el paciente: maltrato al paciente, el profesional no explica en términos accesibles para el usuario, no enfatiza el alcance y las limitaciones del tratamiento en cuestión, no informa del costo y de la relación riesgo/beneficio de la atención (posibilidad de fracaso del procedimiento otorgado), incurre en inexactitudes e imprecisiones, delega responsabilidades y modifica convenios en cuanto a modo, tiempo y costo de la atención brindada. También existe la tendencia a despertar falsas expectativas en los pacientes; el uso y manejo equivocados de materiales y procedimientos no indicados, así como tratamientos simulados, pretensiones clínicamente inalcanzables y técnicas clínicas inexpertas. Es muy probable que esta realidad se repita en el resto de los países de la región.

Para obtener información acerca del conocimiento y la utilización del consentimiento informado en Odontología se realizó una búsqueda bibliográfica en las bases de datos de SciELO y Pub Med y se complementó con una encuesta enviada a odontólogos que trabajan en tres centros de atención dental pertenecientes a la Pontificia Universidad Católica de Chile.

\section{Metodología}

Se utilizaron las palabras clave "consentimiento informado" (informed consent) y "autonomía" (autonomy), las que se relacionaron con la palabra "odontología" (dentistry) por medio de la opción MeSH Database de Pub Med. Después de esto, se seleccionaron los artículos que trataban sobre el uso del consentimiento informado en odontología y que planteaban situaciones similares a las de nuestra realidad.

También se utilizó la biblioteca electrónica SciELO ingresando el término "consentimiento informado".

Una vez revisada la bibliografía encontrada se diseñó una encuesta electrónica con seis preguntas sobre el uso y conocimiento del consentimiento informado, la que fue enviada a 40 odontólogos de tres centros de atención dental relacionados con la Pontificia Universidad Católica de Chile. Uno de ellos atiende a estudiantes de la universidad, otro al personal que trabaja en dicha casa de estudios y el último ofrece atención privada a pacientes. La encuesta fue remitida por correo electrónico y respondida en forma voluntaria y anónima por 26 profesionales, lo que constituye un 65\% de la población estudiada.

\section{Resultados}

Al realizar la búsqueda en Pub Med y SciELO se encontraron algunos estudios sobre el uso del consentimiento informado en Odontología.

Garbin y cols.(4) evaluaron el conocimiento, prácticas y actitudes del cirujano-dentista frente al consentimiento informado y verificaron la presentación y decisión del tratamiento propuesto al paciente y la clase de documento utilizado en la clínica odontológica. Para esto encuestaron a los cirujanos dentistas del municipio de Lucélia, São Paulo, Brasil, y recibieron veinte formularios con respuestas, que equivale al 95,2\% de la población de estudio. Los resultados sugieren que la mayoría de los cirujano-dentistas presentan deficiencias en su conocimiento sobre consentimiento informado, mientras otros desconocen totalmente el tema. Su aplicación no se realiza de manera satisfactoria y una simple autorización es el documento más utilizado en la clínica odontológica.

A partir de sus datos, Miguel y Zemel(5) concluyen que, a pesar de que los conocimientos teóricos sobre la práctica del consentimiento informado son escasos, existe una tendencia significativa a destinar tiempo en la consulta dental para su empleo, especialmente entre los dentistas jóvenes. Los autores consideran imperativo incluir en la malla curricular y en los programas académicos odontológicos principios éticos 
y legales con relación al proceso de consentimiento informado. En su estudio también concluyen que algunas características de los profesionales pueden jugar un rol mayor en la forma como se practica este procedimiento. Por ejemplo, las actitudes frente a malas prácticas o la previa exposición a éstas parecen influir en el comportamiento. Profesionales que conocen bien a un paciente pueden llevar a cabo con él un proceso de consentimiento diferente al que realizan con un paciente menos conocido.

En el trabajo de Mortensen y cols.(6) se plantea que, tanto en la consulta médica como en la dental, los pacientes no comprenden adecuadamente la información entregada durante la discusión del consentimiento, especialmente los de menor educación y bajos ingresos. Por la gran importancia de la aceptación, compromiso y entendimiento del paciente que se someterá a tratamiento de ortodoncia, quisieron estudiar la comprensión de los pacientes y de sus padres antes del tratamiento; para ello, observaron el proceso de obtención del consentimiento informado en una clínica pública dependiente de la Universidad de Washington, con pacientes de diferente origen étnico y de bajos ingresos. Sus resultados indican que, en general, tanto los hijos como los padres recuerdan muy poco de la información entregada por el ortodoncista en cuanto a las razones para realizar el tratamiento, los procedimientos, los riesgos asociados y la responsabilidad que deben asumir los niños durante éste. Esto evidencia que la efectividad de las técnicas usadas para obtener el consentimiento informado en las clínicas públicas no es adecuada, especialmente en las poblaciones de bajos ingresos. Sobre esta base, concluyen que deben hacerse nuevos estudios para mejorar los métodos del proceso de obtención del consentimiento informado en los pacientes que se someterán a ortodoncia, especialmente cuando éstos tengan un bajo nivel de educación $\mathrm{y}$ de ingresos.

Orr y Curtis(7) estudiaron la frecuencia con la que los odontólogos obtienen el consentimiento informado para la administración de anestesia local, un procedimiento de rutina en la práctica dental. Según sus resultados, el consentimiento informado es solicitado más frecuentemente por los odontólogos especialistas y por los que practican la anestesiología como especialidad que por los odontólogos generales. Estos últimos son los que realizan con más frecuencia el procedimiento, no exento de complicaciones, por lo que los autores recomiendan a todos los dentistas obtener el consentimiento informado escrito para la administración de este procedimiento. Enfatizan que su ausencia puede tener implicancias significativas en una demanda por mala práctica posterior a un tratamiento.

Bradford y cols.(8) utilizaron material impreso con las diferentes alternativas de tratamiento a las que podía someterse una pieza dental con problemas pulpares. Incluyeron el número de sesiones, costos y pronóstico de cada opción y entregaron de esta manera la información a sus pacientes. $\mathrm{Al}$ compararlos con un grupo control a los que se les había entregado la información sólo verbalmente, los que recibieron la información impresa tenían mayor conocimiento y la información no aumentaba su ansiedad. Ello en contraste con la opinión de algunos autores, quienes cuestionaban la entrega de más información por una supuesta mayor ansiedad secundaria. Los autores concluyen que es útil el uso de material explicativo impreso para informar a los pacientes.

En la encuesta realizada en la Pontificia Universidad Católica de Chile, ante la pregunta sobre la aplicación del consentimiento informado en su práctica clínica, un $50 \%$ respondió que lo usaba sólo en ciertos procedimientos y sólo el $31 \%$ lo practicaba habitualmente y conocía sus implicancias. El 19\% de los encuestados manifestó que conocían su existencia pero que no lo utilizaban en su práctica clínica. De aquellos que respondieron positivamente al uso del consentimiento informado $(81 \%)$, respecto del tiempo dedicado para esto, un $60 \%$ respondió que le brindaba todo el tiempo que fuera necesario. El resto se distribuye entre los que no le dedican tiempo (16\%), los que le dedican entre 10 y 15 minutos (12\%) y los que dedican menos de 10 minutos (12\%).

La manera de obtener el consentimiento informado se traduce en un documento escrito en el $62 \%$ de los casos, el resto lo obtiene en forma verbal. Un $52 \%$ de los encuestados considera que el proceso de obtención del consentimiento se limita a entregarle al paciente la información sobre el mejor tratamiento. Para un 58\% de los profesionales la finalidad del consentimiento informado es que el paciente conozca y decida la mejor alternativa de tratamiento. Para un $50 \%$ su finalidad es evitar problemas legales.

Al consultar cuándo es importante obtenerlo, un 58\% declara que lo hace antes de realizar cualquier procedi- 
miento. Para el resto, sólo cuando la intervención que se realizará implica un riesgo para el paciente, ante una cirugía o un tratamiento invasivo. Del total de la muestra de profesionales, un $77 \%$ es de sexo masculino y un $23 \%$ femenino.

Respecto de los años de ejercicio profesional, el 46\% declara tener entre 16 y 30 ańos de titulado como odontólogo, el $31 \%$ entre 6 y 15, el 15\% más de 30 ańos de ejercicio profesional y el $8 \%$ entre uno y cinco años.

La mayoría de los odontólogos que respondieron la encuesta se dedican al ejercicio de la odontología general en un $35 \%$, o a la rehabilitación oral en un $42 \%$.

\section{Discusión}

Los datos obtenidos en nuestra encuesta, aunque corresponden a un grupo pequeño de profesionales, permiten observar algunos hechos importantes. Uno es la persistencia de una actitud paternalista por parte de los odontólogos hacia sus pacientes: ellos entregan la información sobre cuál es la mejor alternativa de tratamiento y no perciben el consentimiento informado como un proceso participativo.

Basados en la experiencia internacional, podemos decir que aún hay una fuerte tendencia a considerar el consentimiento informado como una herramienta que protege de problemas legales y reclamos, más que como un proceso en el que se toman las decisiones en forma conjunta y responsable por parte del paciente y el profesional.

La necesidad de realizar un proceso de consentimiento informado es vista en muchos casos como exclusiva para los casos de intervenciones riesgosas, invasivas o previo a cirugías, y no como parte de la toma de decisiones en todos los procedimientos que se llevan a cabo con pacientes.

La necesidad de realizar un proceso para llegar a la obtención del consentimiento informado en el área de la odontología es unánimemente aceptada. Nuestro ordenamiento jurídico, a diferencia de los de gran parte de los países desarrollados y algunos latinoamericanos, carece absolutamente de regulaciones sobre la actividad médica y los derechos de los pacientes. No existe, en efecto, disposición legal alguna que haga una referencia explícita a derechos y deberes entre médico y paciente, ni menos un sistema o estatuto jurídico relativo a los pacientes.

En todo caso, lo anterior no significa que las personas en su condición de pacientes se encuentren totalmente indefensos. Los derechos y deberes de los pacientes, pese a no encontrarse como un cuerpo legal concreto, completo y preciso, se encuentran dispersos en distintos cuerpos legales, reglamentarios y constitucionales. Actualmente se encuentra en tramitación en la Cámara de Diputados de Chile la ley sobre Deberes y Derechos de las Personas en la que se señala que: "Toda persona tiene derecho a ser informada sobre su condición de salud, tratamientos posibles, evolución y pronóstico en forma oportuna y comprensible, de acuerdo a su edad, condición personal o emocional, por el médico, enfermera, matrona u otro profesional tratante, según sea el caso".

El derecho a la información por parte del paciente y su consecuencia, el consentimiento informado, no deben basarse exclusivamente en el cumplimiento de la ley, sino en su fundamento ético irrenunciable: el respeto por la dignidad y la libertad de las personas. No debe convertirse en un instrumento de desconfianza que nos separe del paciente; su objetivo último no es ser exclusivamente un respaldo jurídico para el profesional.

El consentimiento informado es realmente un proceso, resultado de un trabajo permanente de comunicación entre el equipo de salud y el paciente, uno de los fundamentos sobre el cual se establece la relación clínica.

Aunque en odontología aún no es práctica habitual, el ejercicio moderno de la profesión y la valoración del paciente y sus derechos obligan a que el consentimiento informado sea incluido en los procedimientos realizados a los pacientes.

Existe desinformación sobre la forma de uso, utilidad y finalidad del consentimiento informado en odontología, por lo que la práctica de su obtención debe iniciarse desde la formación del odontólogo en las instituciones de educación dental y así lograr un cabal entendimiento de su finalidad e importancia. 


\section{Referencias}

1. Cardozo C, Rodríguez E, Lolas F, Quezada A. Ética y Odontología. Una Introducción. Santiago de Chile: CIEB Universidad de Chile; 2007: 105-114.

2. Lorda PS, Gutiérrez JJ. Consentimiento Informado. Medicina Clínica 2001; 117: 99-106.

3. Triana Estrada J. La ética: Un Problema para el Odontólogo. Acta Bioethica 2006; 12(1): 75-80

4. Garbin CAS, Garbin AJJ, Saliba NA, Zina LG, Gonçalves PE. El consentimiento informado en la clínica odontológica, Acta Odontológica Venezolana 2007; 45(1): 37-43 [SciELO].

5. Miguel R, Zemel M. El Consentimiento Informado en odontólogos residentes de la Facultad de Odontología de la Universidad Nacional de la Plata. Acta Bioethica 2006; 12(1): 81-89 [SciELO].

6. Mortensen MG, Kiyak HA, Omnell L. Patient and parent understanding of informed consent in orthodontics. Am J Orthodontics and Dentofacial Orthopedics 2003; 124: 541-550 [ PUB MED].

7. Orr DL, Curtis WJ. Obtaining written informed consent for the administration of local anesthetic in dentistry. JAm Dent Assoc 2005; 136(11): 1568-1571. [PUB MED].

8. Johnson BR, Schwartz A, Goldberg J, Koerber A. A Chairside Aid for Shared Decision Making in Dentistry: A Randomized Controlled Trial. J Dent Educ 2006; 70: 133-141 [PUB MED].

Recibido: 7 de enero de 2009

Aceptado: 28 de enero de 2009 